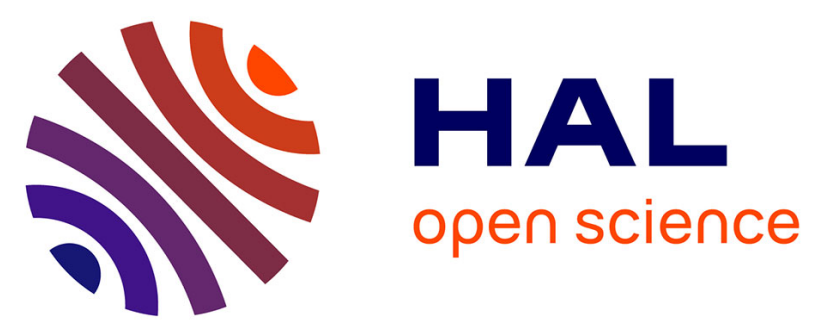

\title{
Analysis and comparison of the results of two ray tracing-based sonic boom propagation codes applied to the SBPW3 test cases
}

Gérald Carrier, Patrice Malbéqui, Pierre-Elie Normand

\section{To cite this version:}

Gérald Carrier, Patrice Malbéqui, Pierre-Elie Normand. Analysis and comparison of the results of two ray tracing-based sonic boom propagation codes applied to the SBPW3 test cases. AIAA Scitech 2021 Forum, Jan 2021, VIRTUAL EVENT, United States. 10.2514/6.2021-0231 • hal-03224232

\section{HAL Id: hal-03224232 \\ https://hal.science/hal-03224232}

Submitted on 11 May 2021

HAL is a multi-disciplinary open access archive for the deposit and dissemination of scientific research documents, whether they are published or not. The documents may come from teaching and research institutions in France or abroad, or from public or private research centers.
L'archive ouverte pluridisciplinaire $\mathbf{H A L}$, est destinée au dépôt et à la diffusion de documents scientifiques de niveau recherche, publiés ou non, émanant des établissements d'enseignement et de recherche français ou étrangers, des laboratoires publics ou privés. 


\title{
Analysis and comparison of the results of two ray tracing-based sonic boom propagation codes applied to the SBPW3 test cases
}

\author{
Gérald Carrier ${ }^{1}$, Patrice Malbéqui ${ }^{2}$ \\ DAAA, ONERA, Paris Saclay University, F-91123, Palaiseau, France \\ Pierre-Elie Normand ${ }^{3}$ \\ Dassault Aviation, Saint-Cloud, 92210, France
}

\begin{abstract}
This paper presents a comparison of the sonic boom propagation results obtained by ONERA and Dassault Aviation for the two test cases of the third AIAA Sonic Boom Prediction Workshop with their two propagation codes, respectively BANGV and DABANG. In addition to the material presented during the workshop, a more in-depth analysis of some ray-tracing results such as the data along the acoustic rays is presented. Such code-to-code comparisons proved to be useful to detect and correct minor coding differences and mistakes that can be present in codes and therefore increase the confidence and validation of these codes.
\end{abstract}

\section{Nomenclature}

$\mathrm{CFD}=$ Computational Fluid Dynamics

$\mathrm{PDE} \quad=$ Partial Derivative Equations

SBPW3 = Third AIAA Sonic Boom Prediction Workshop

\section{Introduction}

In the context of the third AIAA Sonic Boom Prediction Workshop (SBPW3)[6] and of the EU-funded H2020 research project RUMBLE [1] (RegUlation and norM for low sonic Boom LEvels) ONERA and Dassault Aviation have applied two different sonic boom propagation codes, namely the BANGV and DABANG codes, to predict the ground sonic boom signature for several test cases. These two propagation codes are based on a ray-tracing approach (geometrical acoustics theory) accounting for the diffraction effects resulting from the vertical stratification of the temperature, density and wind through the atmosphere and solving along each acoustic rays a non-linear Burgers equation with dissipative effects. The two different cases studied are those of the SBPW3 and involve different near-field pressure signatures representative of low-boom aircraft in steady cruise flight condition, as well as different atmosphere profile representative of real meteorological conditions. This code-to-code comparison exercise enabled a verification of the implementation of the different numerical methods used to solve the same phenomena with similar physical assumptions. The detailed investigation of the discrepancies in results, especially on conditions resulting in the largest differences between the two codes, helped identify their origins and apply corrections to the codes when needed.

The first section of this paper introduces the two ray tracing codes used for these comparisons. Then the test cases of the SBPW3 are briefly summarized in the second section. Finally, the results of the codes for the two test cases are analyzed and discussed in the last section.

\footnotetext{
${ }^{1}$ Research Engineer, Aerodynamics, Aeroelasticity and Acoustics Dept., ONERA, Member AIAA.

${ }^{2}$ Research Engineer, Aerodynamics, Aeroelasticity and Acoustics Dept., ONERA, Member AIAA.

${ }^{3}$ Research Engineer, Aerodynamics Dept., Dassault Aviation, Member AIAA.
} 


\section{Description of the ray propagation codes}

Assuming that the sonic boom sources are characterized in the near field, e.g. by the aerodynamic pressure perturbations extracted from a Computational Fluid Dynamics (CFD) simulation on a cylinder surrounding the aircraft flying at supersonic speed, the sonic boom signature experienced by a fixed observer on the ground can be calculated by mean of a ray-tracing code (Figure 1).

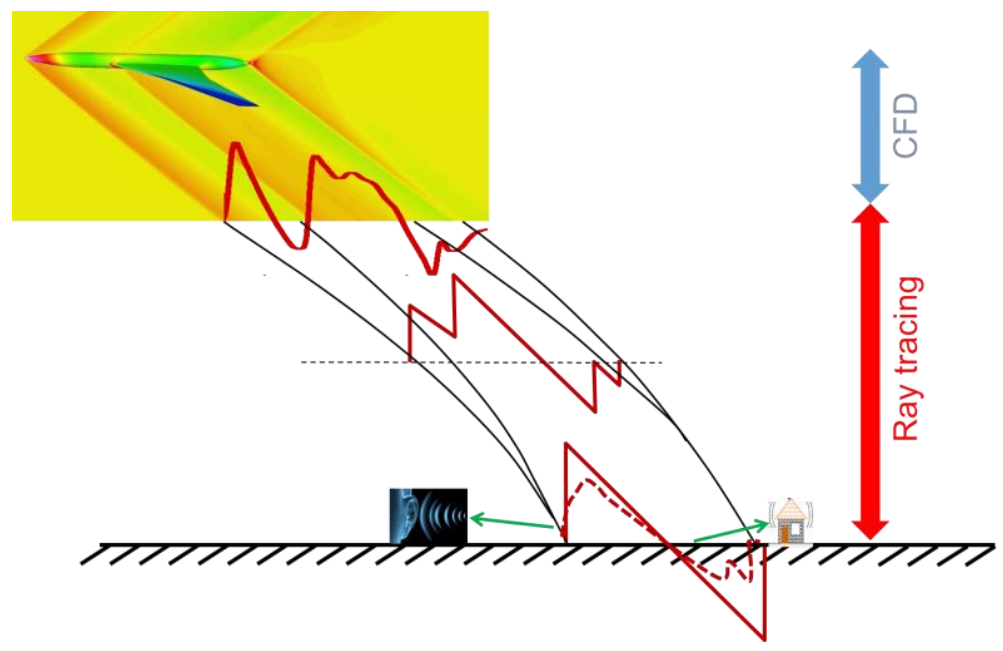

Figure 1 Sonic boom simulation. Near field is calculated by CFD and ground propagated sonic boom is calculated by ray tracing.

The main specificities of the two sonic boom propagation codes BANGV and DABANG based on the geometrical acoustic theory and ray tracing approach are listed below. More details can be found in [2] to [4]. The codes allow for sonic boom propagation under the following assumptions:

- Any shape of the aircraft is possible; the near-field signature can be modeled by the so-called Whitham function or with a cylindrical pressure distribution at a given distance of the aircraft, derived from the CFD or the wind tunnel measurements;

- Various aircraft trajectories can be simulated, such as cruise, maneuver or even acceleration with focusing;

- A stratified meteorology without turbulence (no temporal fluctuations in the media during the propagation) is assumed, with profiles of temperature, wind, relative humidity and density; the profiles are interpolated by cubic splines or piece-wise linear functions;

- A flat ground without absorption is considered but with a reflection coefficient of 1.9.

The two codes are based on the same ray theory, stating that the sound propagating along rays can be determined according to the Fermat principle. This theory is the well-known high frequency approximation, assuming locally plane wave propagation. The amplitude of the wave form is predicted along rays, through the cross-section of the raytube, using the Blokhintsev invariance principle. The numerical implementation follows the formulation derived by Candel [5]. It is based on the integration of a system of 13 Ordinary Differential Equations (ODE) for BANGV and 19 for DABANG: respectively 4 and 6 ODEs to determine the rays, 8 and 12 ODEs to estimate the ray-tube crosssection, and $1 \mathrm{ODE}$ for the nonlinear age variable.

To account for the strong non-linear effects occurring during the propagation in the atmosphere due to the amplitude of the peak overpressures in the sonic boom signal, the codes solve the non-linear dissipative Burgers's equation, governing the distortion of the signal, associated with an appropriate technique to automatically handle the shock position. The acoustical absorption due to the thermos-viscous effects and the molecular relaxation of nitrogen and oxygen molecules is performed using a split-step algorithm. Concerning BANGV, the diffraction effects included in the shadow zone using creeping waves provides a smooth transition of the acoustical pressure field between the geometrical carpet and the shadow zone after cutoff. Focusing of the rays occurs under refraction at low Mach number, an aircraft accelerating or during a sharp turning. For these configurations, the nonlinear Tricomi's equation is solved, where the "N" wave can be transformed into a "U" wave, and the overpressure level can be amplified by a factor up to 5 . 


\section{Description of the two tests cases}

The third Sonic Boom Prediction Workshop (SBPW3) was held in January 2020 during the AIAA SciTech 2020 conference [6]. It included a portion dedicated to the propagation of sonic boom signatures. Two test cases were designed for the purpose of exercising and assessing the capabilities of ray-tracing codes dedicated to the prediction of sonic booms. Participants to the workshop were required to produce results for these two cases and provide their results on the ground for some primary carpet points corresponding to acoustic rays emitted at different specified azimuthal angles and also for the limiting rays, as it is detected by their own code. These results included the coordinates of the impact points of these rays on the ground, the predicted pressure-time signatures at these points and the associated loudness metrics.

The two test cases of the propagation portion of SBPW3 are described in [7]. They differ by the near-field signal corresponding to two different low-boom notional aircraft, by the flight condition (Mach number and altitude) and finally and most importantly by the characteristics of the atmospheres which correspond to vertically stratified profiles of density, temperature, humidity and velocity magnitudes and direction.

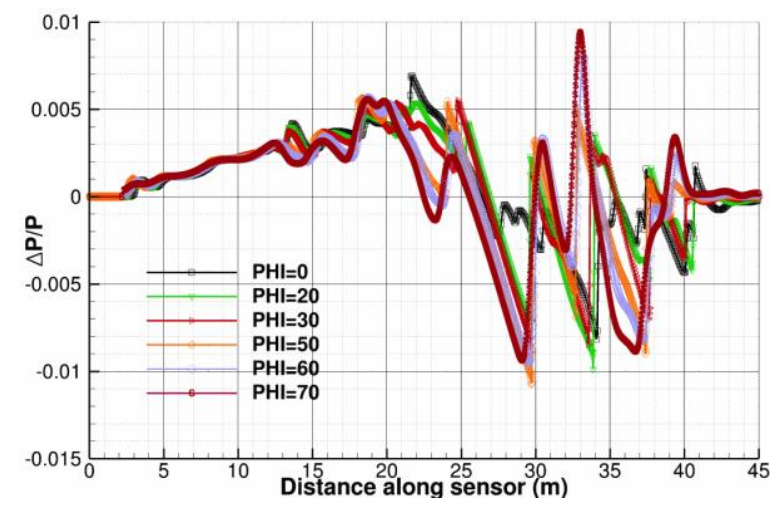

(a) Near field pressures

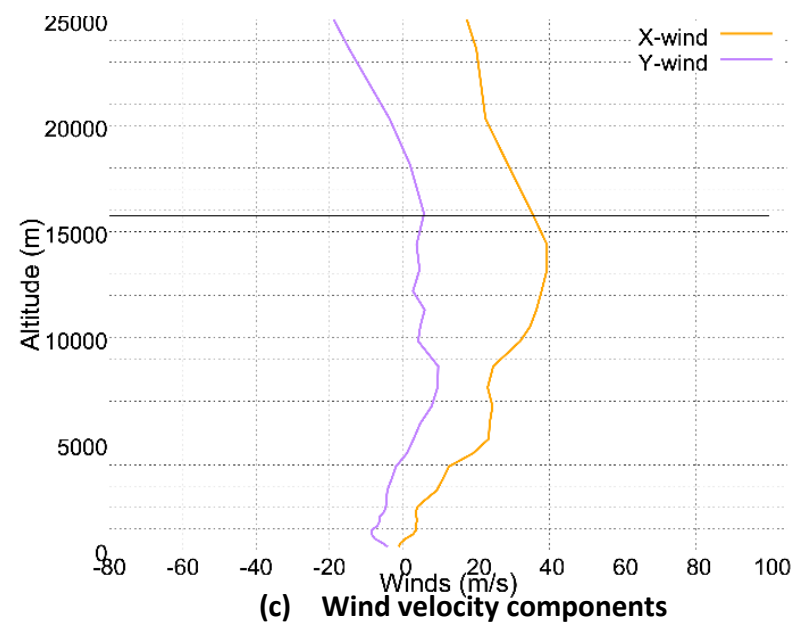

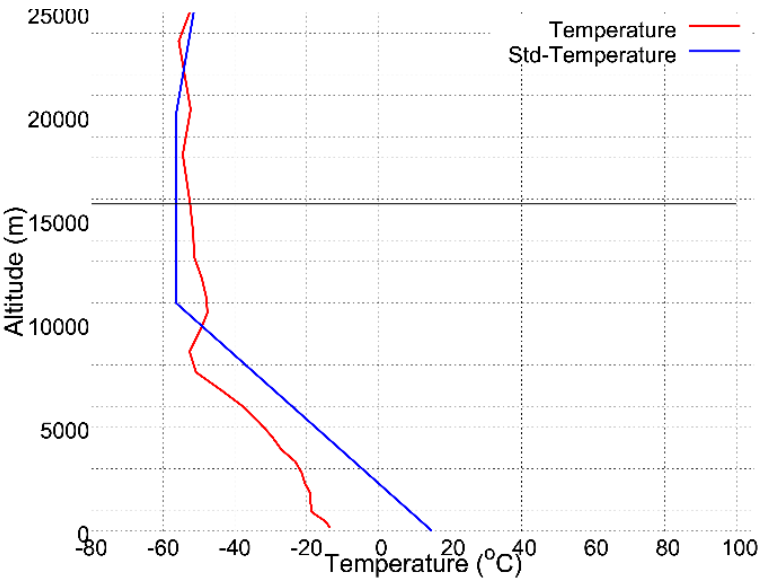

(b) Temperature profile

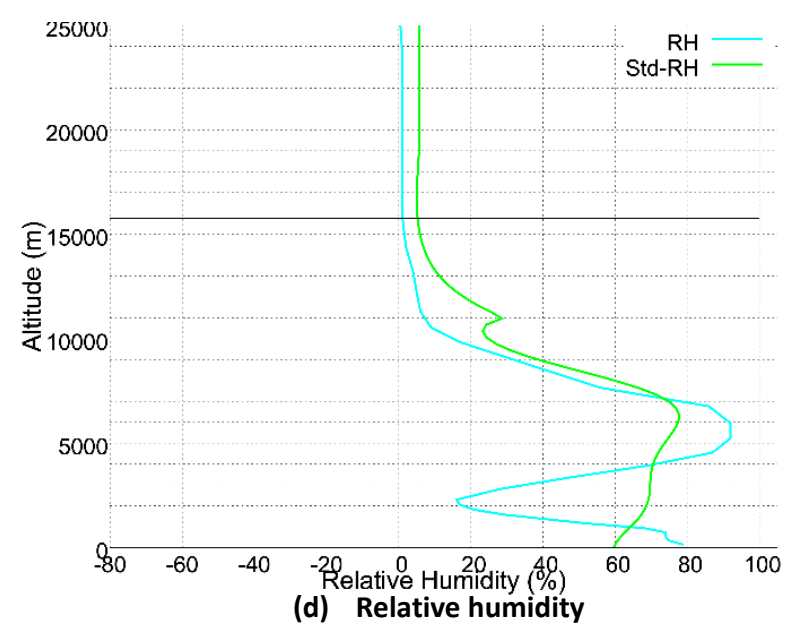

Figure 2 Definition of the first test case (Case1) of SBPW3-propagation portion (extracted from [7]): (a) near field pressures and atmosphere profiles for temperature (b), wind velocity components (c) and relative humidity (d) 


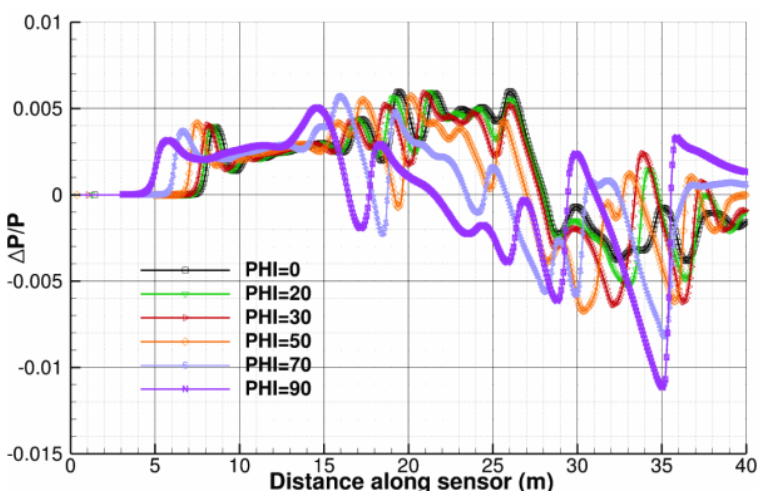

(a) Near field pressures

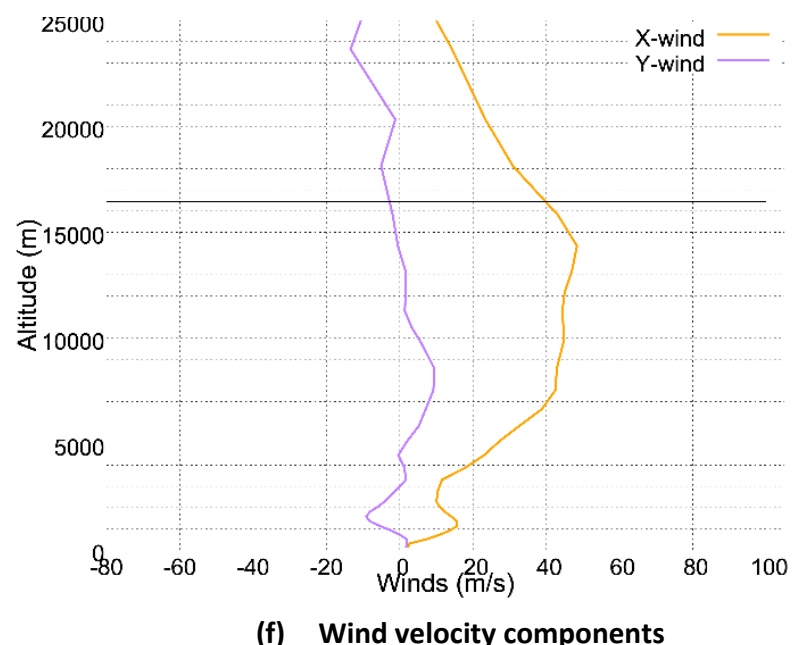

(f) Wind velocity components

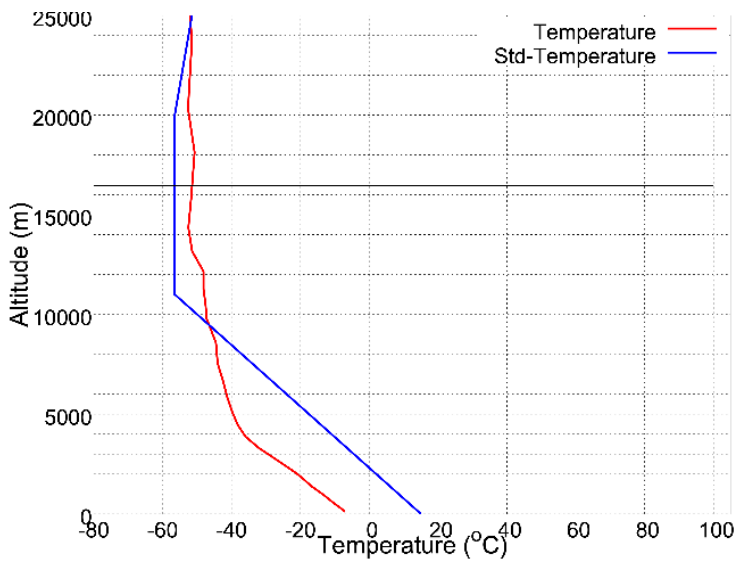

(e) Temperature profile

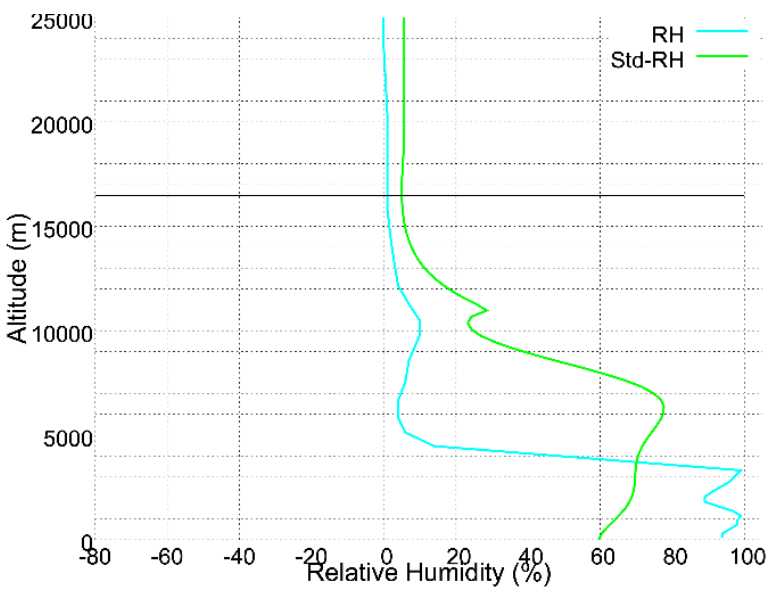

(g) Relative humidity

Figure 3 Definition of the second test case (Case2) of SBPW3-propagation portion (extracted from [7]): (a) near field pressures and atmosphere profiles for temperature (b), wind velocity components (c) and relative humidity (d)

The first test case (Figure 2) corresponds to steady flight conditions at Mach number 1.6 and an altitude of $15.760 \mathrm{~m}$ heading towards the East. It includes an atmosphere profile (distributions of density, temperature and velocity of wind with altitude) chosen on purpose to achieve the maximum range of limiting azimuthal angles between the left and right limiting rays defining the primary carpet. The second test case (Figure 3) corresponds also to steady flight conditions but at a Mach number 1.4, an altitude of $16459.2 \mathrm{~m}$ and heading towards the East. It included an atmosphere profile chosen to produce the widest primary carpet.

\section{Analysis of the results}

\section{A. Results for SBPW3 Case1}

Both DABANG and BANV were applied for the SBPW3 Case1 test case which includes moderate wind effects but a temperature profile that differs significantly from standard atmosphere. Firstly, the predicted ray paths were analyzed and compared between both codes.

The difference in the paths calculated by the two codes for rays emitted at the exact same azimuth angles is negligible as illustrated in left-hand side of Figure 4. The prediction of the limiting rays that define the lateral primary carpet edge are very sensitive to the atmosphere profile, in this case resulting in almost horizontal rays above ground level.

Tableau 1 shows the comparison of the position of the limiting rays on both side of the primary carpet, both in term of the azimuth angle and ground impact location. Although the difference in the prediction of the limiting azimuth 
angles is within $0.2^{\circ}$ between the two codes, this can yield differences in the prediction of the carpet width of more than $10 \mathrm{~km}$. The right-hand side of Figure 4 shows the differences in ray paths for the carpet edge rays.
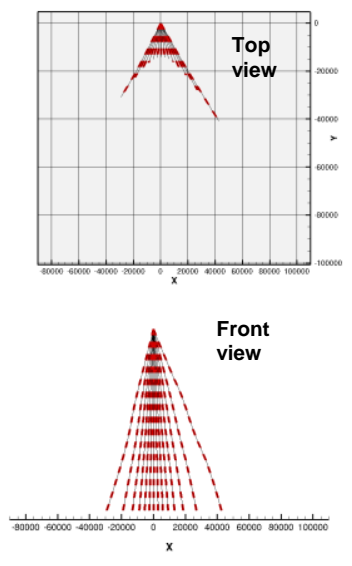
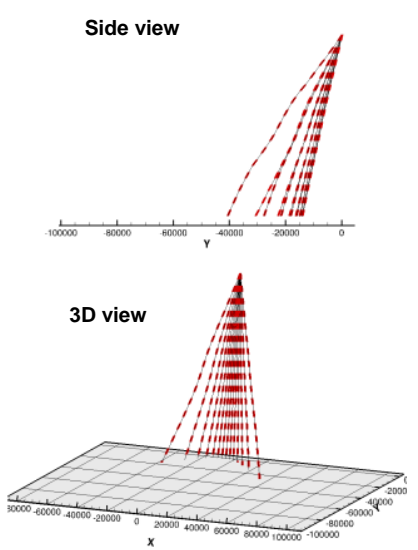
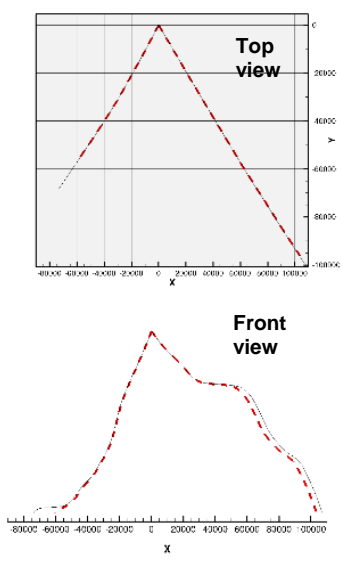
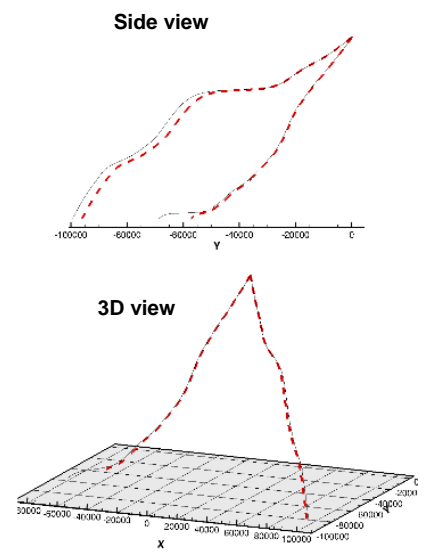

Figure 4 Comparison of ray paths emitted at different azimuthal angles ranging from $-60^{\circ}$ to $60^{\circ}$ (left) and cut-off rays (right) for SBPW3 Case1. . BANGV results in black and DABANG in red.

\begin{tabular}{|c|c|c|c|c|}
\hline Codes & $\begin{array}{c}\text { Lateral Cut-off } \\
\text { Angles (min) }\end{array}$ & $\begin{array}{c}\text { Lateral Cut-off } \\
\text { Angles (max) }\end{array}$ & $\begin{array}{c}\text { Lateral Carpet } \\
\text { Edges (left) }\end{array}$ & $\begin{array}{c}\text { Lateral Carpet } \\
\text { Edges (right) }\end{array}$ \\
\hline BANGV & $-78.41^{\circ}$ & $69.01^{\circ}$ & $106.959 \mathrm{~km}$ & $73.747 \mathrm{~km}$ \\
\hline DABANG & $-78.4^{\circ}$ & $68.8^{\circ}$ & $103.388 \mathrm{~km}$ & $60.252 \mathrm{~km}$ \\
\hline
\end{tabular}

Tableau 1: Comparison of BANGV and DABANG results for the carpet limiting rays data; SBPW3 Case1.

The intermediate variables solved along the rays by the geometric part of the ray solver are compared. Figure 5 shows the results for the ray tube cross-sectional area and Blokhintsev coefficient. An exact match is observed between the two codes (within numerical accuracy limit). Note that the ray tube area is an algebraic value that is calculated in both code by integrating an exact Jacobian of the ray-path. It has a negative sign in this case.

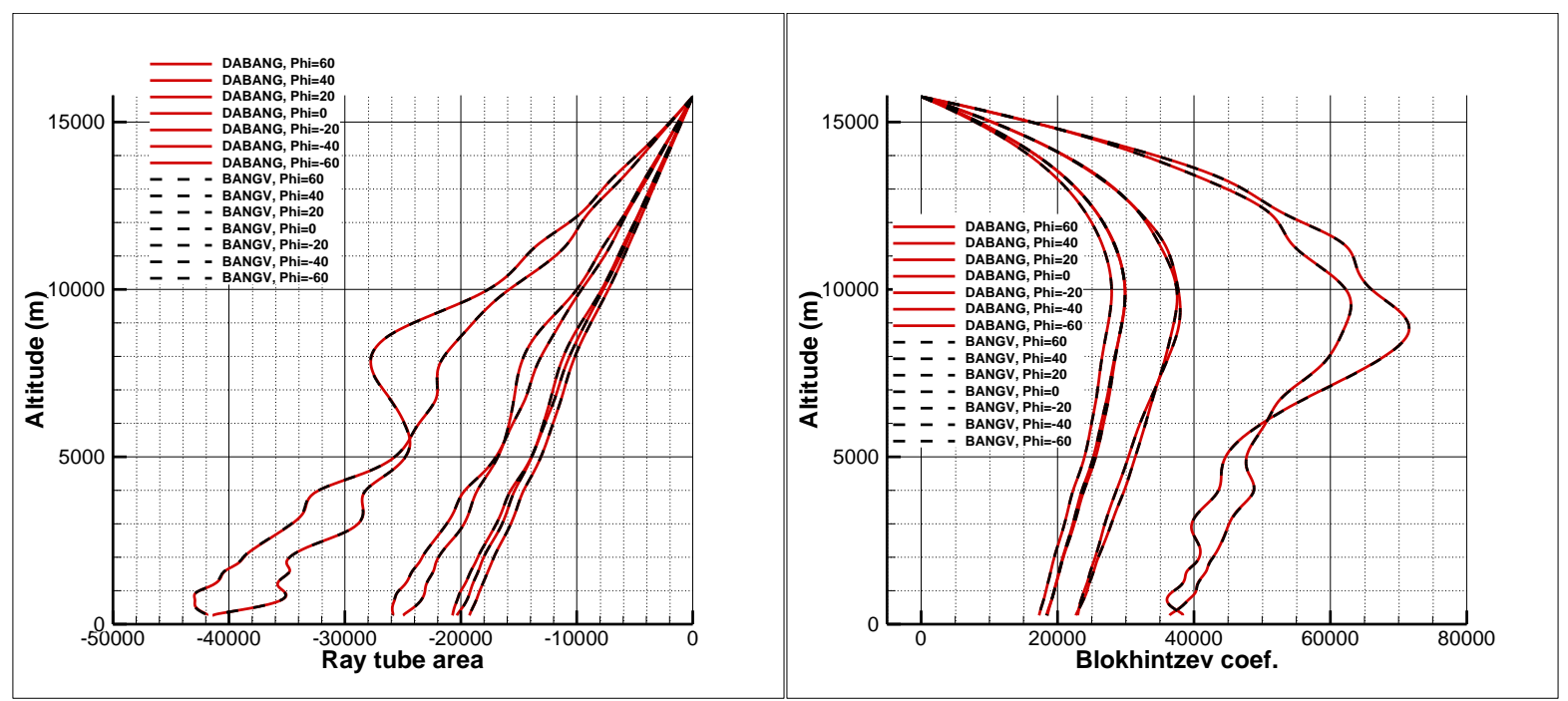

Figure 5 Comparison of ray tube area (left) and Blokhintsev coefficient (right) along the rays emitted at different azimuthal angles ranging from $-60^{\circ}$ to $60^{\circ}$ for SBPW3 Case1. 
The differences in the ground pressure signatures predicted by the two codes are plotted in Figure 6 . With the exception of the signatures at the carpet edge, which exhibit more important differences (due to the difference in the prediction of cut-off angles), all other azimuth angles are very closely predicted with identical duration, shapes and only minor differences in amplitudes and rise time (BANGV predicts slightly lower overpressure amplitudes and slightly higher time rises).

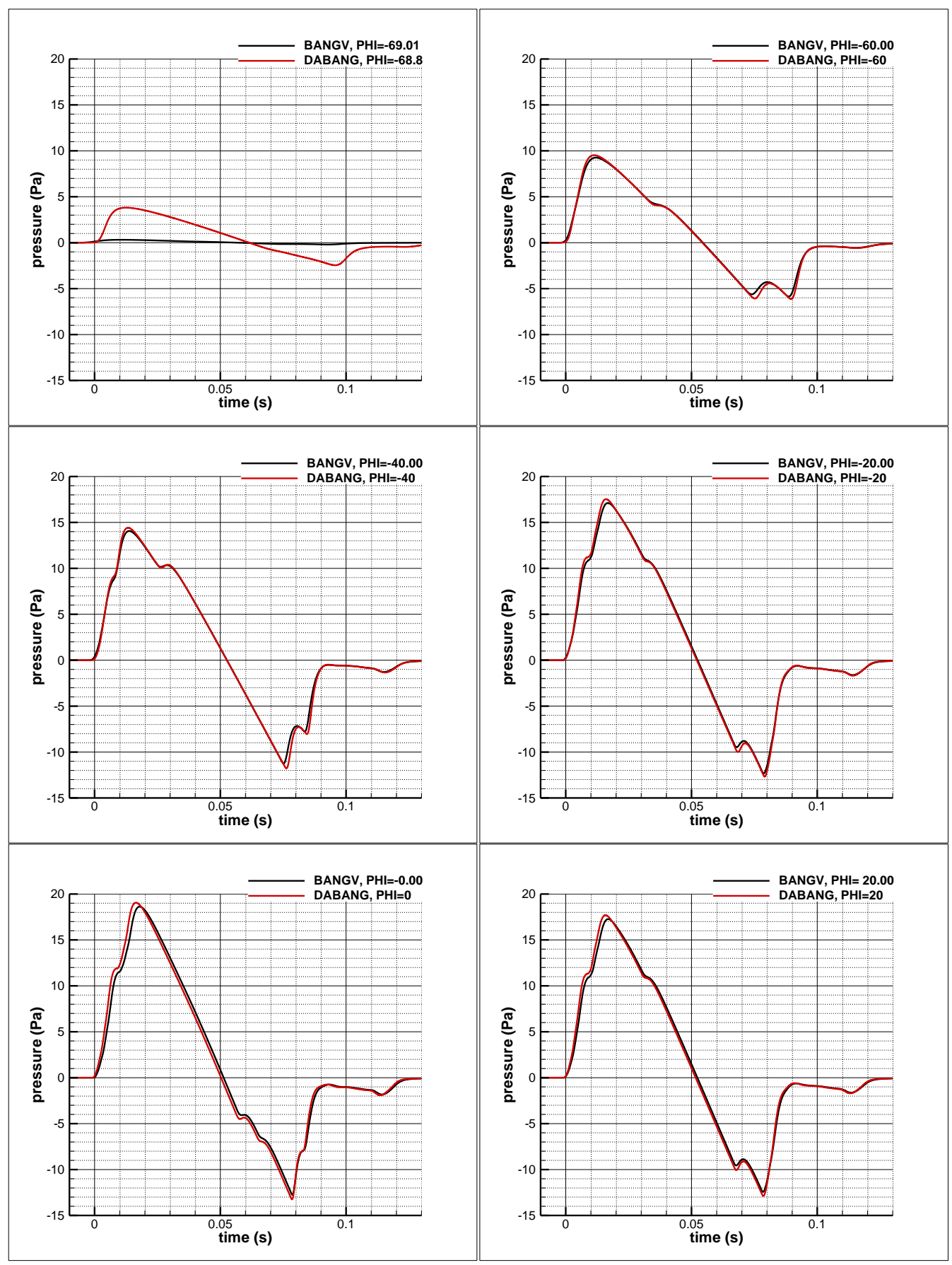




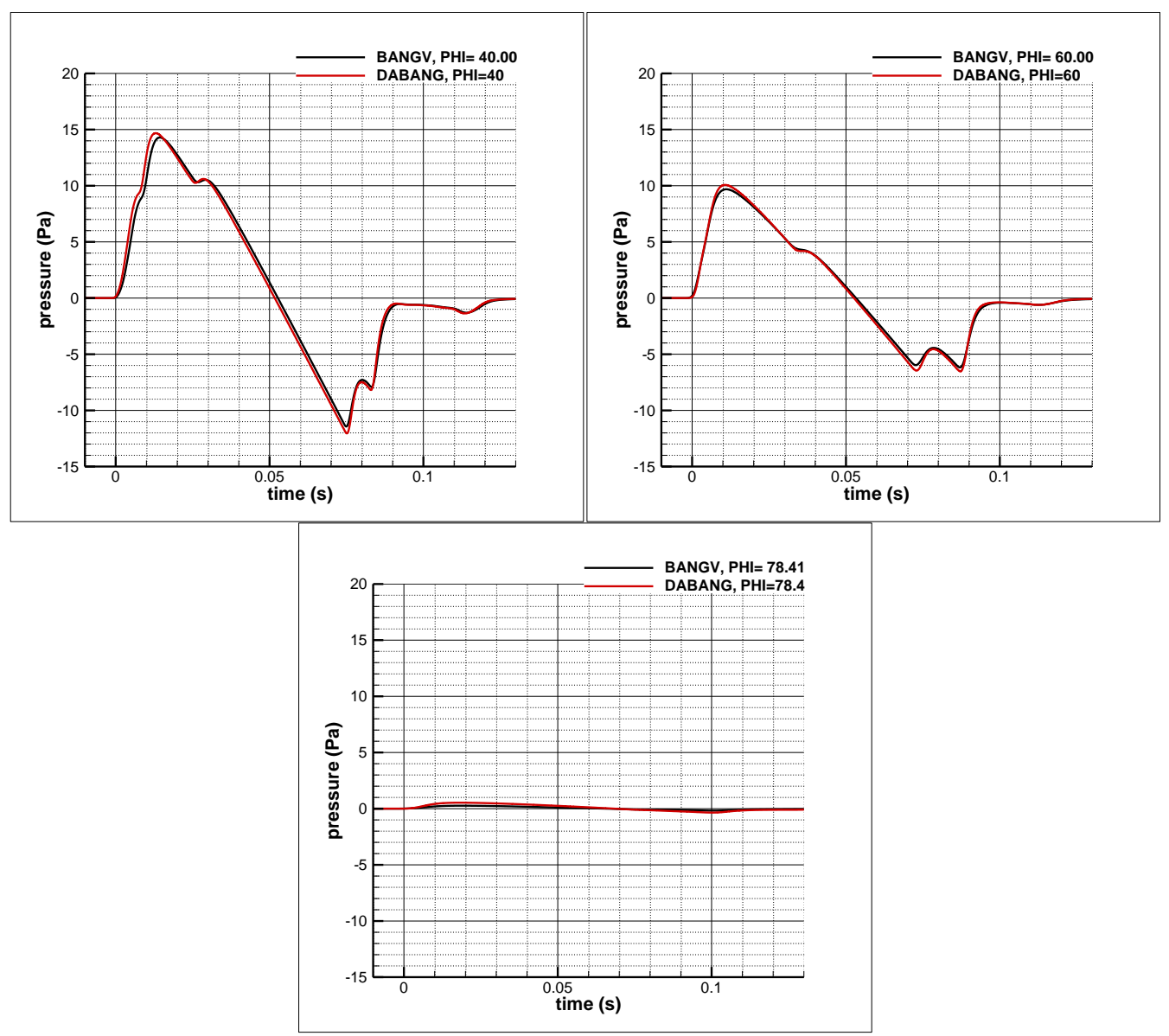

Figure 6 Comparison of ground pressures for rays emitted at different azimuthal angles ranging from $-60^{\circ}$ to $60^{\circ}$ for SBPW3 Case1.

In order to investigate the causes of the remaining differences between the two codes on this test case, results have been reproduced with both codes without taking into account any dissipative or dispersive effects. The motivation was, once the rays' variables matched sufficiently closely, to check that the non-linear effects were similarly predicted by the Burgers equations solvers of the two codes, without introducing the additional complexity of other effects. As shown in Figure 7, the two codes provide nearly identical results without absorption effects, indicating that the remaining differences observed on ground pressures in Figure 6 are mostly due to the way these absorption phenomena are accounted for in the two codes. 


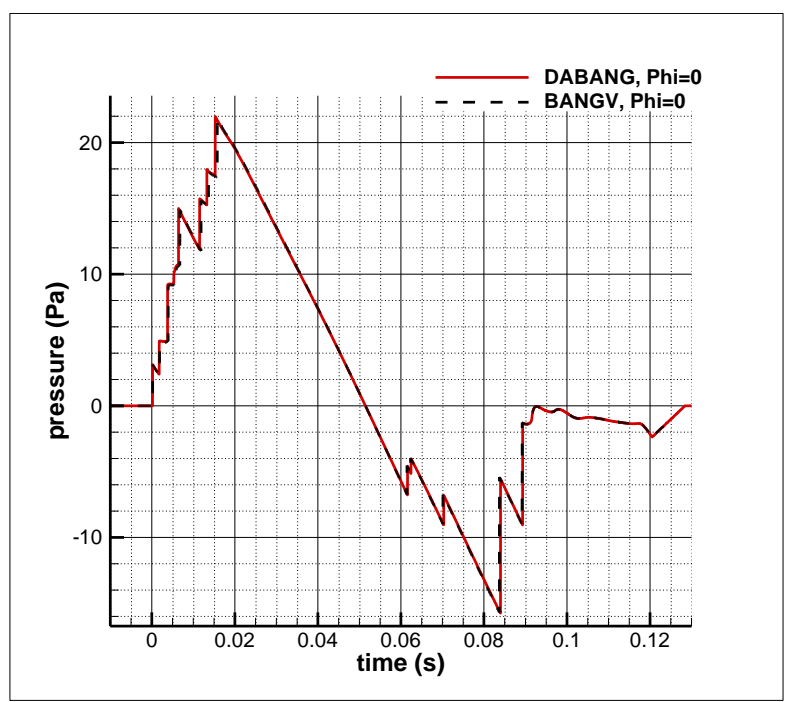

Figure 7 Comparison of ground pressures for ray emitted at zero azimuthal angle for SBPW3 Case1 (without absorption).

\section{B. Results for SBPW3 Case2}

Such comparisons between the DABANG and BANV codes were reproduced for the SBPW3 Case 2 test case which included more severe wind effects. Again, the predicted ray paths were first analyzed and compared between both codes. Figure 8 shows a comparison of the ray paths predicted by both codes in the conditions of Case2. For rays emitted at the same azimuth angle, difference in calculated ray paths are negligible. The detection of the limiting azimuth angles of rays at carpet edge is very sensitive (due to the dichotomy process) to such atmospheres, resulting in limit rays with almost horizontal tangent above the ground (see left hand side of Figure 8). Therefore, the prediction of the cut-off angles and carpet semi-width are slightly different between the two codes as shown in Tableau 2.

The resulting differences in the paths of the rays limiting the carpet (cut-off rays) is observable on right hand side of Figure 8 .
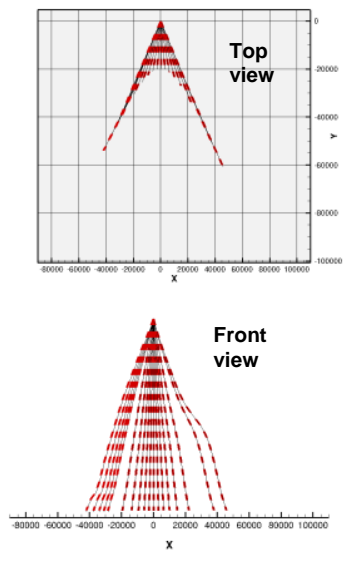
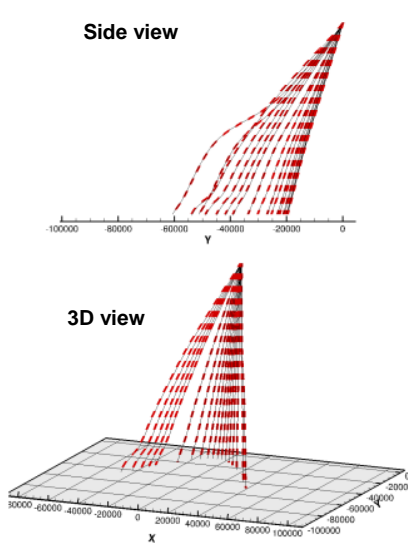
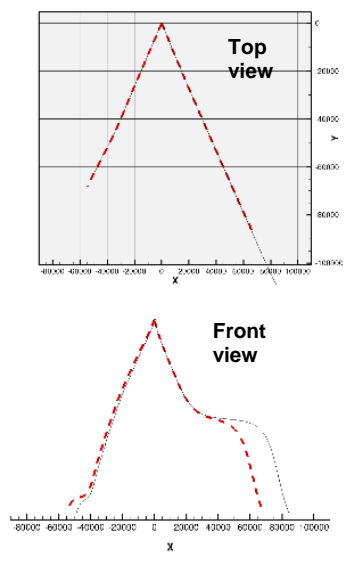
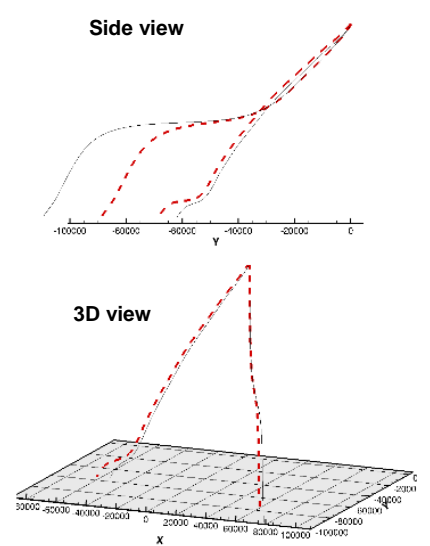

Figure 8 Comparison of ray paths emitted at different azimuthal angles ranging from $-60^{\circ}$ to $60^{\circ}($ left) and cut-off rays (right) for SBPW3 Case2. BANGV results in black and DABANG in red. 


\begin{tabular}{|c|c|c|c|c|}
\hline Codes & $\begin{array}{c}\text { Lateral Cut-off } \\
\text { Angles (min) }\end{array}$ & $\begin{array}{c}\text { Lateral Cut-off } \\
\text { Angles (max) }\end{array}$ & $\begin{array}{c}\text { Lateral Carpet } \\
\text { Edges (left) }\end{array}$ & $\begin{array}{c}\text { Lateral Carpet } \\
\text { Edges (right) }\end{array}$ \\
\hline BANGV & $-63.68^{\circ}$ & $70.07^{\circ}$ & $-84.023 \mathrm{~km}$ & $49.083 \mathrm{~km}$ \\
\hline DABANG & $-63.6^{\circ}$ & $70.6^{\circ}$ & $-67.917 \mathrm{~km}$ & $54.453 \mathrm{~km}$ \\
\hline
\end{tabular}

Tableau 2: Comparison of BANGV and DABANG results for the carpet limiting rays data; SBPW3 Case2.
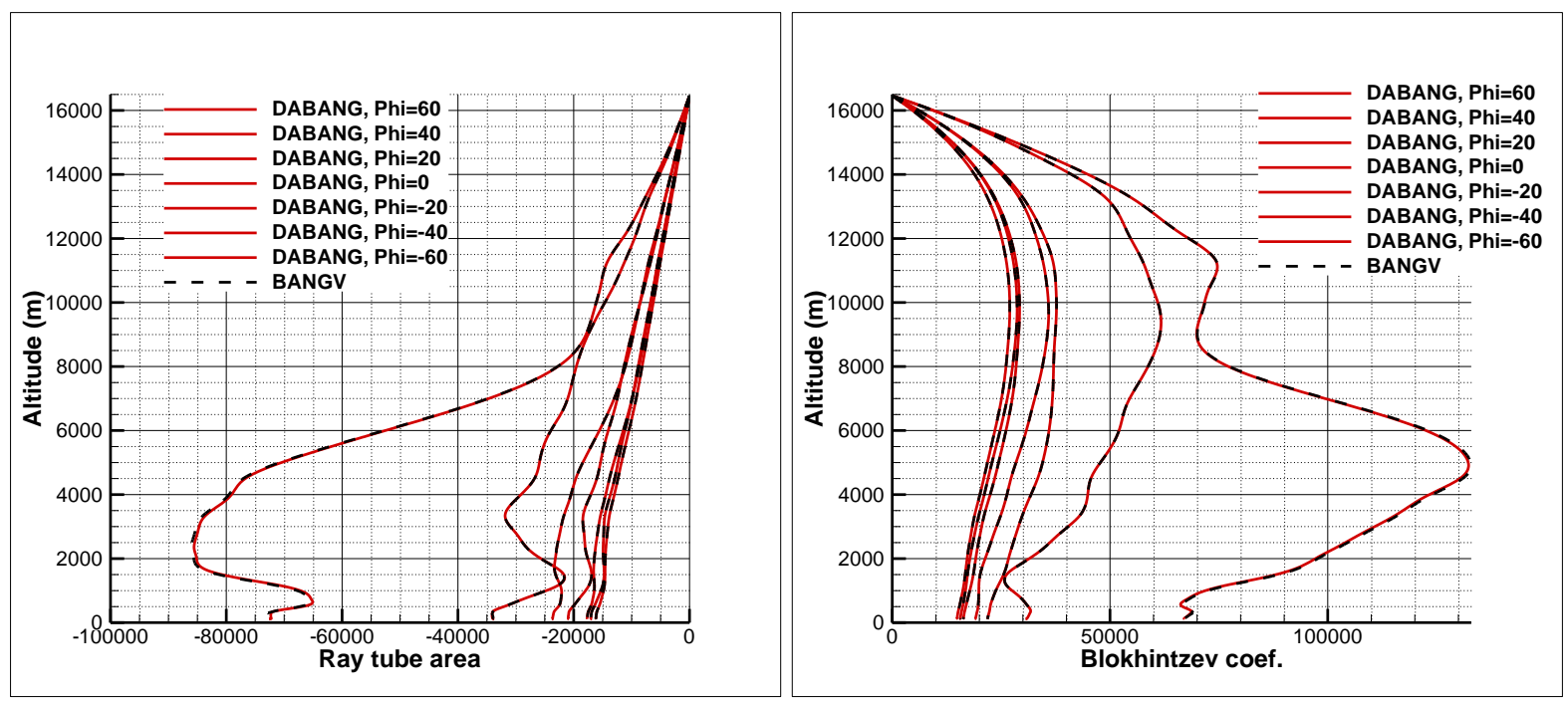

Figure 9 Comparison of ray tube area (left) and Blokhintsev coefficient (right) along the rays emitted at different azimuthal angles ranging from $-60^{\circ}$ to $60^{\circ}$ for SBPW3 Case2.

The differences in the ground pressure signatures predicted by the two codes are plotted in Figure 9. Here again, with the exception of the signatures at the carpet edge which present more important differences (due to the difference in the prediction of cut-off angles), the signatures at all other azimuth angles are very closely predicted with identical duration, shapes and only minor differences in amplitudes (this time BANGV predicts overpressure amplitude slightly higher). 


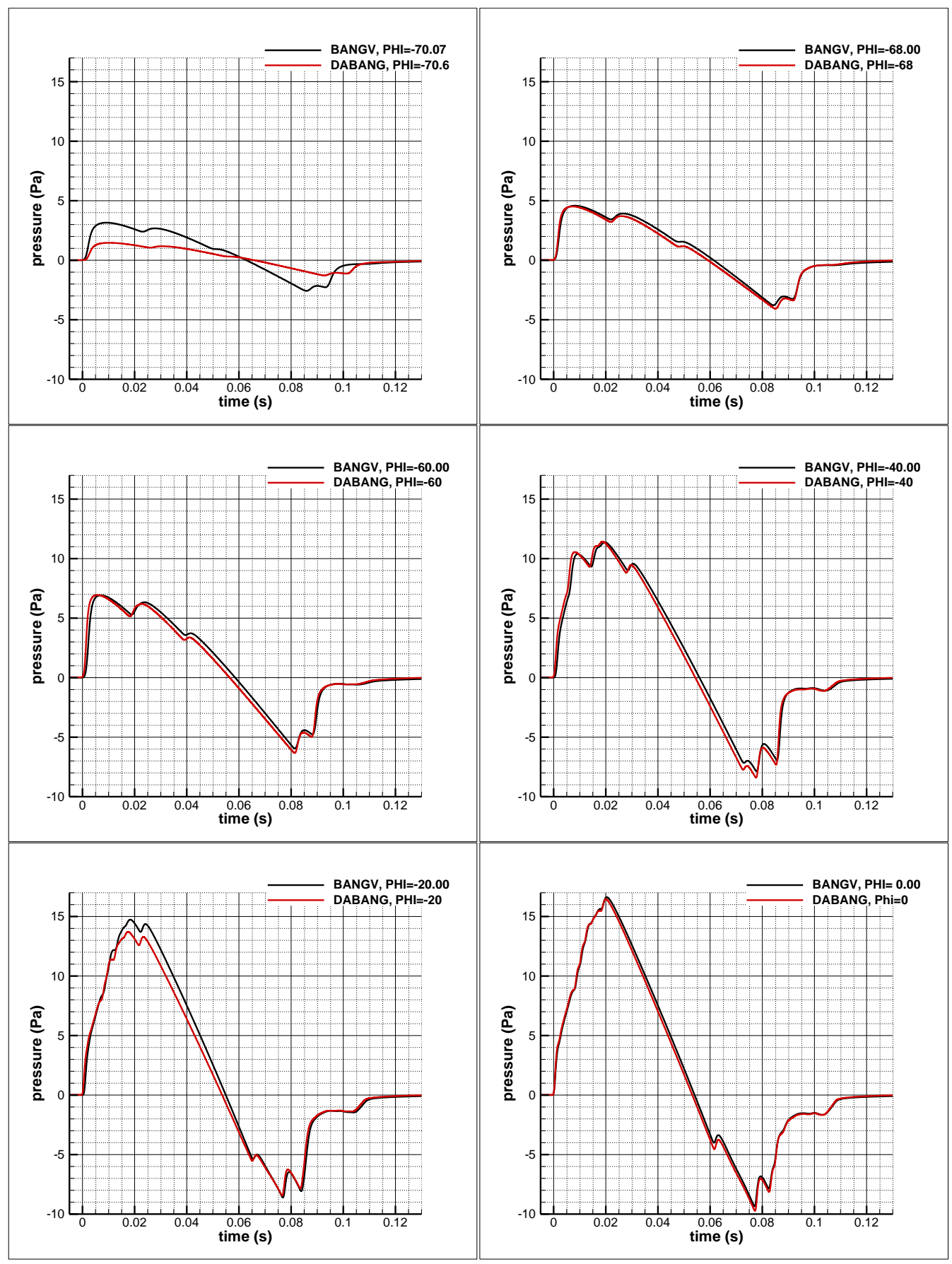




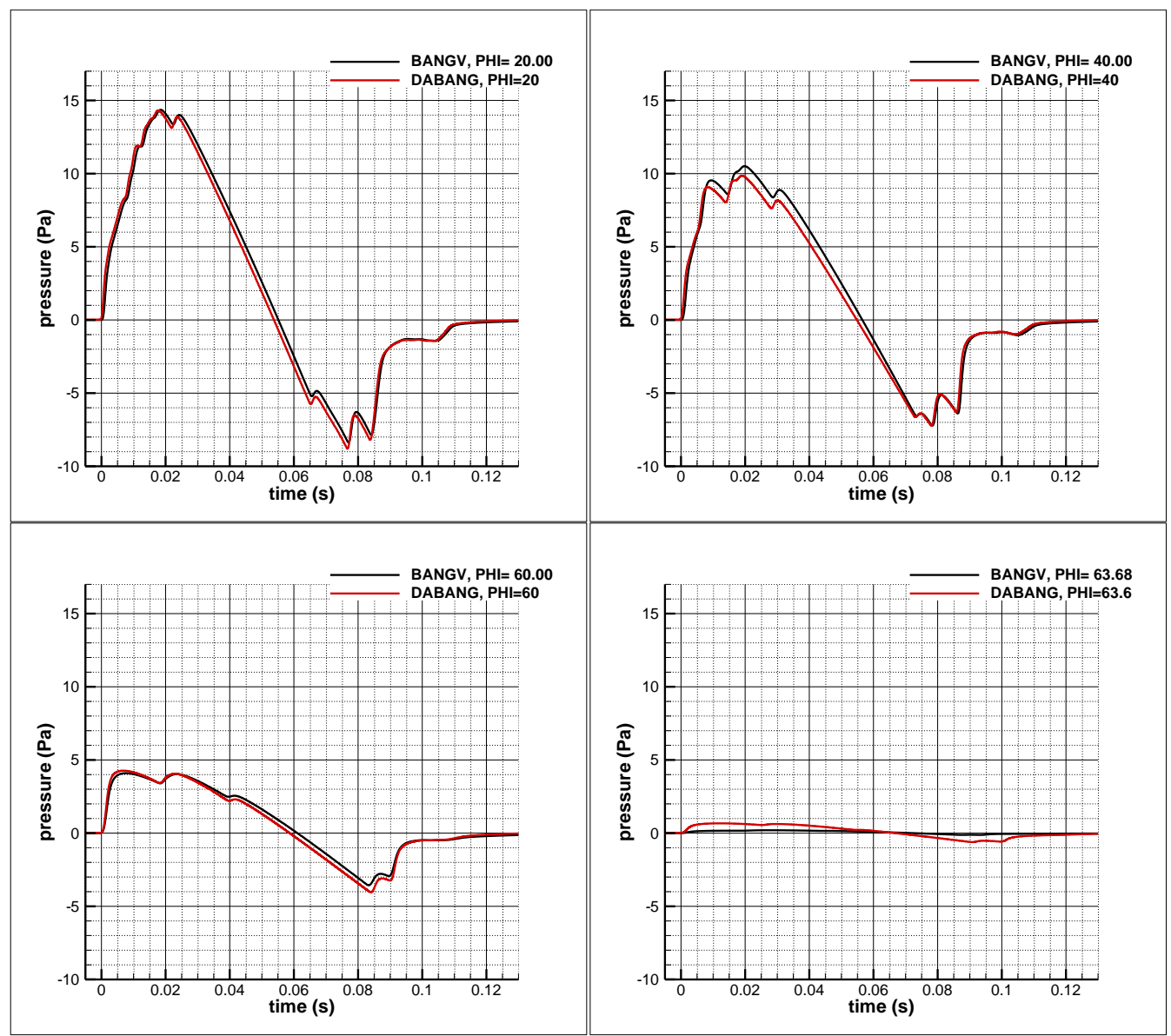

Figure 10 Comparison of ground pressures for rays emitted at different azimuthal angles ranging from $-60^{\circ}$ to $60^{\circ}$ for SBPW3 Case2.

As for Case1, results have been reproduced with both codes without taking into account any dissipative or dispersive effects in order to verify that the remaining differences between the two codes for this Case 2 were also mainly due to the absorption phenomenon. As shown in Figure 11, the two codes provides very close results without absorption effects, confirming on a second case that the remaining differences observed on ground pressures in Figure 10 are mostly due to the way the absorption phenomena is accounted for in the code. 


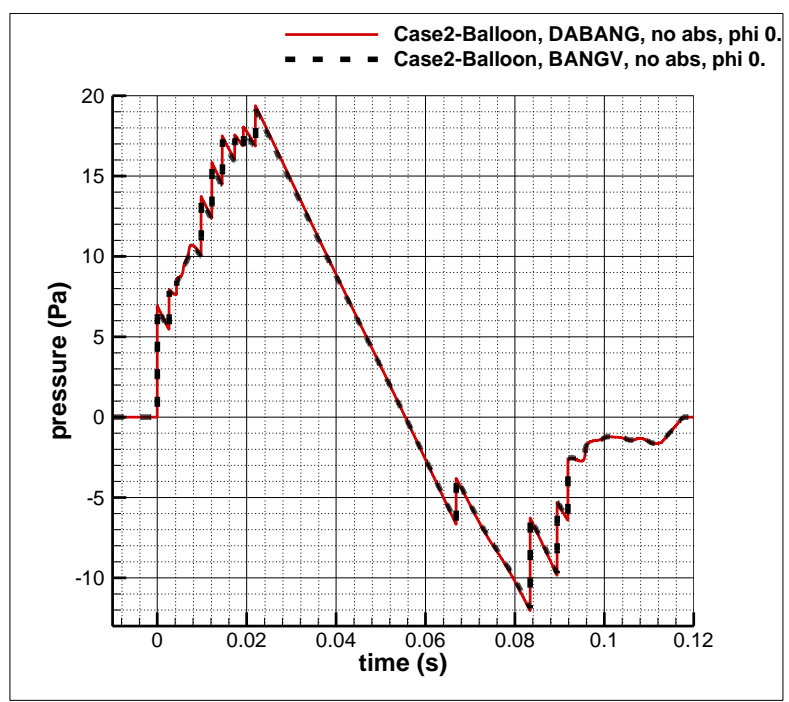

Figure 11 Comparison of ground pressures for ray emitted at zero azimuthal angle for SBPW3 Case2 (without absorption).

\section{Conclusion}

A verification of two sonic boom propagation codes based on acoustic ray tracing, namely the BANGV code used by ONERA and the DABANG code used by Dassault Aviation is presented on the basis of the two test cases of the propagation portion of AIAA SBPW3. A detailed analysis of the observed differences along the rays enabled to identify some differences and correct some discrepancies present in the codes. After those corrections, the two codes provided very similar and consistent results for all aspects except those related to the account of absorption. Further verifications of how these dissipative and dispersive effects are accounted for by the two codes are ongoing.

\section{Acknowledgments}

The work presented in this paper has been partly funded by the RUMBLE[1] project that has received funding from the European Union's Horizon 2020 research and innovation program under grant agreement No 769896.

\section{References}

[1] https://rumble-project.eu/i/sites/default/files/Downloads/RUMBLE_brochure.pdf

[2] Dagrau, F., "Simulation de la propagation du bang sonique: de la CFD à l'acoustique non linéaire," Ph. D. Thesis, Sorbonne Université Paris 6 (Université Pierre et Marie Curie), 2009.

[3] Marchiano, R., Coulouvrat, F. and Grenon, R. "Numerical simulation of shock waves focusing at fold caustics, with application to sonic boom," J. Acoust. Soc. Am., Vol. 114, pp.1758-1771, 2003.

[4] Anonymous, BANGV: the Sonic Boom Software, User's Guide, Version 4.0, December 2008.

[5] Candel, S.: "Numerical solution of conservation equations arising in linear wave theory: application to aeroacoustics," $J$. Fluid Mech., Vol. 83, pp. 465-493, 1977.

[6] https://lbpw.larc.nasa.gov/sbpw3/

[7] Rallabhandi, S. and Loubeau, A. "Summary of Propagation Cases of the Third AIAA Sonic Boom Prediction Workshop", to be published 